\title{
Non-unitary evolution of quantum logics
}

\author{
Sebastian Fortin, Federico Holik and Leonardo Vanni
}

\begin{abstract}
In this work we present a dynamical approach to quantum logics. By changing the standard formalism of quantum mechanics to allow non-Hermitian operators as generators of time evolution, we address the question of how can logics evolve in time. In this way, we describe formally how a non-Boolean algebra may become a Boolean one under certain conditions. We present some simple models which illustrate this transition and develop a new quantum logical formalism based in complex spectral resolutions, a notion that we introduce in order to cope with the temporal aspect of the logical structure of quantum theory.
\end{abstract}

\section{Introduction}

Non-Hermitian Hamiltonians [1] find many applications in diverse areas of physics such as for example, optics [2,3], solid state physics [4], decoherence[5], the quantum to classical limit, and final equilibrium [6]. Decoherence and relaxation times can be defined using non-unitary evolutions, pole theory, and non-Hermitic Hamiltonians $[6,15]$. In this work we study the logical properties of quantum systems under the evolution given by a Non-Hermitian Hamiltonian, in order to provide a quantum logical description of the classical limit.

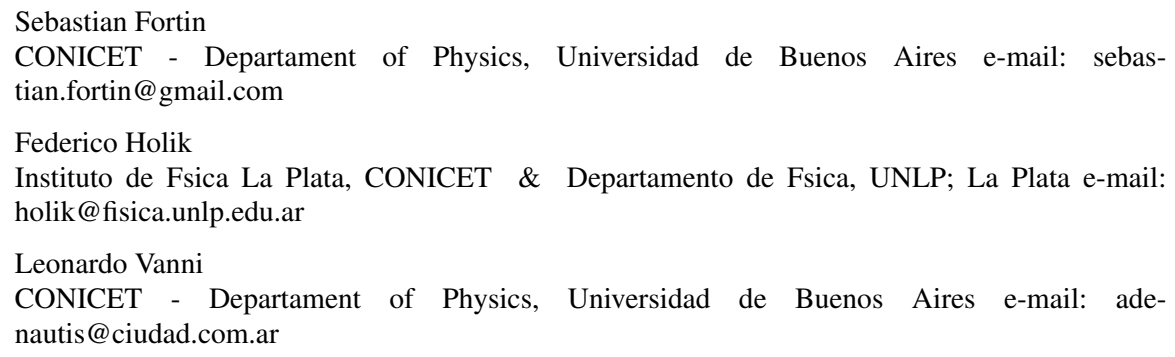


The rigorous formulation of quantum mechanics was achieved after a series of papers by von Neumann, Jordan, Hilbert and Nordheim [16]. Projection operators play a key role in the axiomatization, and this is related to the spectral decomposition theorem $[17,18]$, which associates a projection valued measure to any quantum observable represented by a self adjoint operator [19]. The set of projection operators can be endowed with an orthomodular (non-Boolean) lattice structure [20,22] and was named quantum logic [21], in contrast with the distributive structure of classical propositional systems [23]. This approach allows to compare quantum and classical systems by putting them in a common mathematical framework [24]. The quantum logical approach can be also used to provide a solid axiomatic foundation for quantum mechanics, and to explain in an operational way many of important features of the Hilbert space formalism [24, 28]. But it turns out that the quantumlogical approach has not addressed, up to now, the wholly important problem of characterizing the dynamical transformation in the logic of a system undergoing a decoherence process [29] and the reaching of the classical limit. As was shown in [31], the study of the classical limit offers the possibility of describing the transition from the quantum logic of a quantum system to the Boolean logic characteristic of a classical one. We will continue this line of research in this work by using nonHermitian time evolutions.

In the standard approach to decoherence [32, 29], the classical limit is reached by the effect of the interaction between the environment and the system. An important model is that of a quantum system interacting with a heat bath of harmonic oscillators. In certain cases, the study of these examples gives rise to quantum Langevin equations (see for example [30]). But there exists an alternative approach, which allows for the possibility of studying the classical limit in terms of the evolution of mean values of relevant sets of observables [34, 41]. According to this framework, it is possible to make an analytical continuation on the energy variable into the lower complex half-plane for any possible Hamiltonian of the system. In general, poles will be found $[42,5]$, and they can be used to define all possible nonunitary decaying modes with their respective characteristic decaying times, which are proportional to the inverse of the imaginary part of the poles ${ }^{1}$. Poles are complex eigenvalues of the non-Hermitian Hamiltonian $H_{\text {eff }}$ which governs the time evolution of the system. Using these characteristic times it is possible to deduce the relaxation time, which turns out to be the inverse of the imaginary part of the pole which lies closest to the real axis. Therefore, it is the largest characteristic time. It is also possible to compute the decoherence time, which turns out to be a function of the imaginary part of the poles and the initial conditions of the system.

The complete description of a quantum system involves non-commutative operators: in the standard approach to quantum mechanics, these are generated by the set of bounded operators $\mathscr{B}(\mathscr{H})$ on a Hilbert space $\mathscr{H}[17,23]$. As a consequence, the lattice of quantum properties will be non-distributive [43, 23]. On the other hand, for classical systems, onservables are represented by functions over phase space and form a commutative algebra; thus, classical properties are distributive [20, 23].

\footnotetext{
${ }^{1}$ We do not consider the Khalfin mode since it has an extremely long decaying time $[47,48]$
} 
According to recent works $[45,46]$, there are certain quantum systems such that, while initially the commutator between two operators can be different from zero, it may tend to zero under the action of certain time evolution operators. In other words, non-Boolean lattices can become Boolean under the action of special time evolutions. On the basis of this observation, in this work, we study the impact of a non unitary evolution on the logical structure of the system by continuing previous works [31]. That is, we study how the logical structure of quantum properties corresponding to relevant observables, becomes essentially Boolean by using an algebraic approach [17].

The paper is organized as follows. In Section 2 we review the standard quantum logical formalism. In Section 3 we review the Schrödinger and Heisenberg pictures in the standard formalism of quantum mechanics and show that they are not suitable for describing a dynamical interpretation of the logical structure. Next, in Section 4, we review the non-Hermitian Hamiltonians approach to quantum theory and show how it can be used to describe the dynamic of logics provided that the Heisenberg picture is used. In Sections 5 and 6 we present concrete examples of this behavior, the first one with only two different characteristic times, and the other with many of them. We summarize the discussion in Section 7 and then present a novel formalism for a dynamical quantum logical approach, based on a generalization of the notion of projective valued measure to the field of complex numbers. Finally, in Section 8 we present our conclusions.

\section{Classical and quantum logics}

The state of a classical particle $S$ is completely determined by its position $\mathbf{q}$ and momentum $\mathbf{p}$. Thus, the state can be considered as point $(\mathbf{p}, \mathbf{q})$ in phase space $\mathbb{R}^{6}$. On the other hand, physical observables are represented as functions over phase space. As an example, consider the energy of the particle $E(\mathbf{p}, \mathbf{q})$. If we now consider an empirically verifiable proposition such as "the energy of the system lies in the interval $(a, b)$ ", we can represent it as the set of states of the system which make that proposition true. Similarly, any empirically verifiable property $O$ can be naturally represented as a set of points $\mathscr{O}$ in phase space, i.e., $\mathscr{O} \subset \mathbb{R}^{6}$. This representation allows to determine if a physical system possesses a given physical property or not: the particle possesses a given property if and only the point $(\mathbf{p}, \mathbf{q})$ representing the state of the particle is included in the set representing that property.

With this assignment between properties and subsets of state space, the logical structure of classical properties gets connected with set theoretical operations. In this way, the conjunction " $\wedge$ " of two assertions represented as subsets of phase space can be described as a set theoretical intersection " $\cap$ "; the disjunction " $\vee$ " as a set theoretical union " $\cup$ ", and the negation " $\neg$ " as the set theoretical complement "(... $)^{c}$ ". A partial order relation " $\leq$ " (understood as a sort of implication) is given by set theoretical inclusion " $\subseteq$ ". For technical reasons it is reasonable to restrict to measurable sets of phase space (this is strongly connected with the necessity of 
computing mean values and probabilities of events) [25, 26]. As is well known, subsets of a given set, endowed with the logical operations described above form a Boolean algebra [23]. A distinctive feature of a Boolean algebra is the distributive law, which asserts that for any propositions $a$ and $b$, we have

$$
a=(a \wedge b) \vee(a \wedge \neg b)
$$

This is just one of the many properties of Boolean logic, and we put it into the spot, because it will allow us to illustrate the differences with the quantum case.

In quantum mechanics the state of a particle is represented by a trace class positive Hermitian operator of trace one, usually called density matrix. Pure states can be represented by one dimensional projection operators or equivalently, as density matrices $\rho$ such that $\rho^{2}=\rho$ [17]. In order to establish the algebraic structure underlying the logical propositions associated to a quantum system, let us take a deeper look into the formal structure of quantum mechanics. Physical observables are represented mathematically as self adjoint operators acting on a Hilbert space. What allows physicists to make this connection? The answer lies in the spectral theorem $[17,18]$, which allows to associate to each self adjoint operator $A$ a projective valued measure (PVM), defined as a function from the Borel sets into the set of projection operators of the Hilbert space satisfying

$$
\begin{array}{r}
P: B(\mathbb{R}) \rightarrow \mathscr{P}(\mathscr{H}) \\
\text { such that } \\
P(\emptyset)=0 \\
P(\mathbb{R})=\mathbf{1} \\
P\left(\cup_{j}\left(B_{j}\right)\right)=\sum_{j} P\left(B_{j}\right),
\end{array}
$$

for any disjoint denumerable family $B_{j}$. Also,

$$
P\left(B^{c}\right)=\mathbf{1}-P(B)=(P(B))^{\perp}
$$

The spectral decomposition theorem allows to put self adjoint operators and PVMs in a one to one correspondence. Despite the mathematical technicalities, these results allow to provide a very clear operational interpretation for observables represented as self adjoint operators as follows. Given an observable $A$, consider the proposition "the value of $A$ lies in the interval $(a, b)$ " (notice that all empirically testable propositions in quantum mechanics are of this form). Using the PVM associated to $A$ (which we call $P_{A}$ ), the real line interval $(a, b)$ is mapped to a projection operator $P_{A}(a, b)$. If the state of the system is described by the density matrix $\rho$, the probability of obtaining the property represented by $P_{A}(a, b)$ when measuring $A$ is given by the Born rule [49]

$$
p\left(P_{A}(a, b)\right)=\operatorname{tr}\left(\rho P_{A}(a, b)\right)
$$


In this way, the above mentioned proposition is naturally described by a projection operator. This can be done for any observable and any associated proposition. Thus, empirically testable propositions in quantum mechanics are represented by the set of projection operators of the Hilbert space. Denote this set by $\mathscr{P}(\mathscr{H})$. As is well known, there is a one to one correspondence between the elements of $\mathscr{P}(\mathscr{H})$ and the set of closed subspaces in the Hilbert space (see for example, Chapter 4.2 of Reference [17]). Thus, we will represent empirical propositions in quantum mechanics interchangeably as closed subspaces or as projection operators. Similarly to classical mechanics, the conjunction of two propositions can be represented as a subspace intersection. In symbols: the conjunction " $\wedge$ " of two propositions represented by closed subspaces $\mathbb{S}$ and $\mathbb{T}$ is given by the subspace intersection $\mathbb{S} \cap \mathbb{T}$ (the intersection of two closed subspaces is always a closed subspace). But alike the classical case, it is not possible to take the disjunction " $\mathrm{V}$ " as the union of subspaces: the union of two subspaces will not be in general subspace, and thus, will not be a valid proposition. The solution to this riddle is to represent the disjunction as the closure of the direct sum of the two given subspaces. Implication will be naturally represented by subspace inclusion $(\mathbb{S} \leq \mathbb{T}$ if and only if $\mathbb{S} \subseteq \mathbb{T}$ ), and negation will be represented by taking the orthogonal complement (with respect to the Hilbert product): $\neg(\mathbb{S})=(\mathbb{S})^{\perp}$. The main difference with classical mechanics relies in the fact that instead of a collection of subsets of a set (and their canonical operations), we now have a set of closed subspaces of a Hilbert space. In this context, it is important to remark that the set theoretical union and the direct sum of subspaces are very different operations; a similar remark holds for the negation as set theoretical complement and the negation as orthogonal complement of subspaces. Thus, it is to be expected to find important differences between the quantum and classical propositional structures. Indeed, it is immediate to check that the distributive law is no longer valid for arbitrary quantum properties $a$ and $b$; in fact, we generally have the inequality

$$
a \leq(a \wedge b) \vee(a \wedge \neg b)
$$

If the above inequality is strict, it is said that we have incompatible properties. From the mathematical point of view, this is a direct consequence of the noncommutativity of the observables involved. Let us take a look at the formal structure of this with more detail. In Reference [23] (Chapter 1, Definition 1.1.3) two observables $A$ and $B$ are said to be compatible if and only if, for every Borel sets $\Delta$ and $\Gamma$, the projection operators $P_{A}(\Delta)$ and $P_{B}(\Gamma)$ satisfy:

$$
P_{A}(\Delta) P_{B}(\Gamma)=P_{A}(\Delta) P_{B}(\Gamma)
$$

The above Equation implies that the sub-quantum logic generated by the set of projections (empirical propositions) associated to $A$ and $B$ form a commutative subquantum logic. But it is well known that a sub-quantum logic is commutative if and only if it is a distributive lattice (Cf. for example, Proposition 4.16 of Reference [17]; see also Theorem 3.1.2 in Section 3 of [23]). And we will also have that, 
if $[A, B] \neq 0$, then there will exist propositions associated to $A$ and $B$ that will not satisfy the distributive equality.

\section{Schrödinger and Heisenberg representations}

Quantum mechanical dynamics is governed by the time evolution operator

$$
U=\exp ^{-i H t}
$$

where $H$ is the Hamiltonian of the system. As is well known, it is possible to understand such an evolution from two different perspectives: Heisenberg and Schrödinger pictures. In the latter one, a physical system has associated an initial state $\rho_{0}$, and any physical magnitude will have associated a self adjoint operator $O$ which remains constant in time. In this case (and assuming that there is no interaction with the environment or any measuring apparatus), the state of the system evolves obeying the following equation

$$
\rho(t)=U \rho_{0} U^{\dagger}
$$

On the other hand, in the Heisenberg representation, the physical system has associated a state $\rho$ which remains constant in time, while any physical magnitude has associated an initial self adjoint operator $\hat{O}_{0}$ which changes in time according to the equation

$$
O(t)=U^{\dagger} O_{0} U
$$

In this way, we have two equivalent formulations of quantum mechanics. The equivalence has its roots in the fact that mean values of physical observables are coincident for each representation

$$
\langle O\rangle_{\rho(t)}=\langle O(t)\rangle_{\rho}
$$

In this way, the empirical content of each representation is the same.

From the point of view of the Schrödinger representation, the commutator between two observables is a constant in time: $\left[O_{1}, O_{2}\right]=O_{1} O_{2}-O_{2} O_{1}$. This means that in this representation, the compatibility of two observables is a synchronic relation: two observables are compatible or incompatible independently of the temporal evolution. Because of these reasons, if we are trying to study the diachronic character of the logic associated to a quantum system, this representation is not suitable. Notwithstanding, the mean value of an observable can change during time. But, as the Hamiltonian is an Hermitian operator, then, any evolution is unitary. In particular, the mean value of the commutator obeys the unitary time evolution equation

$$
\left\langle\left[O_{1}, O_{2}\right]\right\rangle_{\rho(t)}=\left\langle U^{\dagger}\left[O_{1}, O_{2}\right] U\right\rangle_{\rho_{0}}
$$


From the point of view of the Heisenberg representation we have that, as the observables evolve, the commutator evolves in a unitary way. If we call $C$ to the operator representing the commutator between $O_{1}$ and $O_{2}$, then

$$
\left[O_{1}(t), O_{2}(t)\right]=C(t)=U^{\dagger} C_{0} U
$$

In this case, given that the evolution is unitary, it is possible to show that if $C_{0} \neq 0 \Longrightarrow C(t) \neq 0$, and on the other hand, $C_{0}=0 \Longrightarrow C(t)=0$. This means that if two operators are compatible/non-compatible at the beginning, they will remain compatible/non-compatible under the effect of unitary time evolution.

The above considerations show that both the Schrödinger and the Heisenberg representations, if restricted to evolutions modeled by Hermitian self adjoint operators (and thus, unitary evolutions), forces us to a synchronic logic. Notice that a similar analysis could be made for the approach to open quantum system dynamics in the Heisenberg picture [30], but due to reasons of space, we will address this question elsewhere. In this paper we aim to represent time evolution of logics; thus, a natural choice is to change the setting a little bit and use non-Hermitian evolution operators. We follow this strategy below presenting some examples first.

\section{Quantum mechanics with non-Hermitian Hamiltonians in the Heisenberg representation}

In the standard formulation of quantum mechanics the Hamiltonian is a self adjoint operator, and as such, its eigenvalues are real numbers. If we relax this restriction, we win some generality and consequently, a richer dynamics can be described. Indeed, the approach to quantum mechanics based on non-Hermitian operators uses this freedom to obtain a non-unitary evolution [8]. According to the Brussels school, if we consider a non-Hermitian Hamiltonian with complex eigenvalues $z_{n}$, then, it is reasonable to describe it using the formula

$$
H=\sum_{n} z_{n}\left|z_{n}\right\rangle\left\langle\tilde{z}_{n}\right|
$$

where $\left|z_{n}\right\rangle$ is an eigenvector of $H$ which, in certain cases, may no longer belong to the traditional Hilbert space, but to the more general rigged Hilbert space. $\left|z_{n}\right\rangle$ is the Gamov vector which belongs to the space $\Phi_{+}^{\times}$of the Gelfand triplet $\Phi_{+}^{\times} \supset \mathscr{H} \supset \Phi_{+}$, and $\left\langle\tilde{z}_{n}\right| \in \Phi_{+}$(see $[50,55]$ ). We will denote by $\mathscr{H}_{\mathscr{R}}$ to the rigged Hilbert space associated to $\mathscr{H}$.

The complex energy $z_{n}$ possesses a real part $\omega_{n}$ and an imaginary part which we conveniently write as $-\frac{1}{2} \gamma_{n}$. In this way $z_{n}=\omega_{n}-i \frac{1}{2} \gamma_{n}$. The techniques for computing observable quantities in the non-Hermitian setting is similar to that of standard quantum mechanics. The Hamiltonian is introduced in the master equation and the equation is solved. For the case of the time evolution of an eigenstate $\left|z_{n}\right\rangle$ of $\hat{H}$ we obtain a solution analog to that of the standard formalism 


$$
\left|z_{n}(t)\right\rangle=e^{-i \hat{H} t}\left|z_{n}\right\rangle=e^{-i \omega_{n} t} e^{-\frac{1}{2} \gamma_{n} t}\left|z_{n}\right\rangle
$$

But we can now clearly see that a time decreasing exponential factor $\exp ^{-\frac{1}{2} \gamma_{n} t}$ comes into stage. This mathematical property allows to study certain physical phenomena for which decaying rates play a central role in a natural way. Some examples of this are the reach of the mechanical equilibrium, quantum decoherence and decaying rates of unstable particles. As is well known, some of these physical processes can be described using open systems interacting with the environment, as for example, the einselection approach to decoherence. But the approach to quantum mechanics based on non-Hermitian operators offers a natural perspective in which these phenomena can be thought of as intrinsic. This alternative offers some computational advantages for certain examples, and at the same time, poses interesting philosophical discussions [31].

As in Section 3, we adopt the Heisenberg representation where we can compute the evolution of the commutator between two observables

$$
\left[O_{1}(t), O_{2}(t)\right]=C(t)
$$

In this case, given that the evolution is in general non-unitary, the commutator also evolves in a non-unitary way. Some authors (see for example $[46,45]$ ) studied examples of decoherence in the Heisenberg representation. In particular, there are some examples for which the initial commutator between two observables is different from zero. According to what we exposed in Section 3, this means that we are dealing with incompatible properties, and as such, it is not possible to measure both observables simultaneously. Notwithstanding, due to the action of decoherence, the final commutator is zero.

$$
C(0) \neq 0 \longrightarrow C(t \rightarrow \infty)=0
$$

Our proposal consists in interpreting this process in the following manner: two incompatible properties become compatible. In this work we propose this line of thought to study the time evolution of the logical properties of physical systems in time. In other words, we propose a dynamical perspective of the quantum logical approach. As we will show, this is not only a technical perspective, but involves a radical modification of the logics associated to physical systems: time decaying rates will be included in the propositional system.

It is important to remark at this point that the adoption of non-Hermitian operators may imply the existence of the reverse process:

$$
C(0)=0 \longrightarrow C(t \rightarrow \infty) \neq 0
$$

If two observables commute at the beginning of the process, they may become noncommutative as time evolves.

From the quantum logical point of view, these results are very important because they imply that the logical properties associated to a physical system may change in a qualitative way. An example of this is the case of the distributive inequality sat- 
isfied by non-compatible quantum properties. As we have seen, such an inequality is a consequence of the non-commutativity of their associated observables. If the evolution is such that a couple of observables transition from the non-commutative to the commutative case, then the distributive inequality becomes an equality.

$$
\begin{aligned}
& a \leq(a \wedge b) \vee(a \wedge \neg b) \\
& \quad \Downarrow \\
& a=(a \wedge b) \vee(a \wedge \neg b)
\end{aligned}
$$

In order to interpret this fact, it is possible to imagine a very simple case in which the two properties are represented each one by a vector. To illustrate, let us suppose that a quantum system has associated the following Hamiltonian

$$
H=\omega|\omega\rangle\left\langle\tilde{\omega}\left|-\frac{i}{2} \gamma\right| \gamma\right\rangle\langle\tilde{\gamma}|
$$

where $\omega$ is a real energy and $\gamma$ is an imaginary energy related to the decaying rate of that mode. On the other hand, let us consider an observable of the form

$$
O=\frac{1}{2}\left[\left(o_{1}+o_{2}\right)|\omega\rangle\left\langle\tilde{\omega}\left|+\left(o_{1}-o_{2}\right)\right| \omega\right\rangle\left\langle\tilde{\gamma}\left|+\left(o_{1}-o_{2}\right)\right| \gamma\right\rangle\left\langle\tilde{\omega}\left|+\left(o_{1}+o_{2}\right)\right| \gamma\right\rangle\langle\tilde{\gamma}|\right]
$$

where $o_{1}$ and $o_{2}$ are the eigenvalues of the given observable. In this case, the commutator between $\hat{H}$ and $\hat{O}$ evolves in such a way that

$$
[H(t), O(t)] \propto e^{-\gamma t}
$$

In the above expression we can see that the imaginary part of the energy appears in the commutator as the inverse of the characteristic time in an exponential. In this case, for times much bigger than the characteristic time, we can assume that the commutator is zero and that it remains unchanged after that. Then, the commutator between the Hamiltonian and the observable $O$ - which was initially different from zero- vanishes, and thus, the two properties, which were non-compatible at the beginning, become compatible ones. In this case, the evolution of the observables can be interpreted in the following way: the angle between the vectors tends to be smaller when time increases. As far as it is different from zero, the inequality remains, but in the infinite time limit the angle goes to zero, and then, the corresponding observables become commutative. As a consequence, we recover distributivity and a Boolean algebra. 


\section{An example with two different characteristic times}

The simple example presented in the previous Section has the virtue of clarifying the mechanism through which a pair of initially non-commuting observables becomes commutative. But it does not offer too many aspects to question further. The case with more than one characteristic time may offer an interesting variant. Let us suppose then that we have a quantum system with observables $O_{A}, O_{B}$ and $O_{C}$ such that

$$
C_{A-B}(t)=\left[O_{A}(t), O_{B}(t)\right] \propto e^{-\gamma_{1} t} \text { and } C_{A-C}(t)=\left[O_{A}(t), O_{C}(t)\right] \propto e^{-\gamma_{2} t}
$$

If, starting from this point we define the observable $O_{D}=O_{A}+O_{B}+O_{C}$, then we have that

$$
C_{A-D}(t)=\left[O_{A}(t), O_{D}(t)\right]=\left[O_{A}(t), O_{B}(t)\right]+\left[O_{A}(t), O_{C}(t)\right]
$$

follows an evolution in two steps. In order to simplify the description with regard to the non-distributivity we define the functions $f(a, b)=a$ and $g(a, b)=(a \wedge b) \vee$ $(a \wedge \neg b)$. In this way, the equality of Eqn. (1) can be written as $f(a, b)=g(a, b)$ and the inequality of expression (3) can be written as $f(a, b) \leq g(a, b)$. Then we have that:

1. At the beginning, the commutators are different from zero: $C_{D-A}(t) \neq 0, C_{D-B}(t) \neq$ 0 and $C_{D-C}(t) \neq 0$. This means that $O_{D^{-}} O_{A}, O_{D^{-}} O_{B}$ and $O_{D^{-}} O_{C}$ are incompatible. Thus, physical properties $a, b, c$ and $d$ associated to them, will also be incompatible. In this way we have the distributive inequality for the three cases.

$$
f(d, a) \leq g(d, a) \quad f(d, b) \leq g(d, b) \quad f(b, c) \leq g(b, c)
$$

2. After the passage of the first characteristic time $t_{1}=\gamma_{1}^{-1}$, the first term of (21) disappears, but the commutator between $\hat{O}_{A}$ and $\hat{O}_{D}$ remains non-vanishing. This means that a part of the algebra became commutative but the other one did not.

$$
f(d, a) \leq g(d, a) \quad f(d, b)=g(d, b) \quad f(b, c) \leq g(b, c)
$$

3. Finally, after the second characteristic time $t_{2}=\gamma_{2}^{-1}$, the last term of Eqn. (21) disappears, and the commutator between $O_{A}$ and $O_{D}$ vanishes. In this way, the remaining part of the algebra becomes distributive:

$$
f(d, a)=g(d, a) \quad f(d, b)=g(d, b) \quad f(b, c)=g(b, c)
$$

The above example shows that the non-unitary evolution transforms incompatible observables in a complex manner. This is just an example, and physical systems of interest may have associated many different characteristic times. For example, in the model of an harmonic oscillator interacting with a bath of harmonic oscillators, we will find infinitely many characteristic times [56]. This situation offers a much 
more complex dynamic of logics that we will address in future works. But we will give an example in the following Section.

\section{Many different characteristic times}

In reference [5] we have presented a formalism based on finding the poles of the analytic extension of the mean values. In this paper we consider a system with a Hamiltonian that can be divided in two parts:

$$
H=H_{0}+V
$$

$H_{0}$ is supposed to be a known hermitian Hamiltonian (the free Hamiltonian): we know its eigenvalues and eigenvectors. On the other hand $V$ is a perturbation. The total Hamiltonian $H$ is a perturbed Hamiltonian and we can use perturbation theory. This theory tells us that there is a relation between the eigenvectors of the perturbed Hamiltonian $|\tilde{\omega}\rangle$ and the eigenvectors of the non-perturbed Hamiltonian $|\omega\rangle$. This relation is given by the Lippmann-Schwinger equations. To the first order, the equations are [51]

$$
\begin{aligned}
& \langle\psi \mid \tilde{\omega}\rangle=\langle\psi \mid \omega\rangle+\left\langle\psi\left|\frac{1}{\omega+i 0-H} V\right| \omega\right\rangle \\
& \langle\tilde{\omega} \mid \psi\rangle=\langle\omega \mid \psi\rangle+\left\langle\tilde{\omega}\left|\frac{1}{\omega+i 0-H} V\right| \psi\right\rangle
\end{aligned}
$$

To solve these equations we need to go to the complex plane and to compute poles and residues. These poles were deeply studied by the Brussels school led by Prigogine (see [57, 60]). The poles can be interpreted as complex energies of the perturbed system. Then, it is possible to build an effective Hamiltonian and we can compute the evolution of the mean values. The result is that the imaginary parts of the poles appear in these mean values as the characteristic times of the exponentials. The mean value can be split in three parts: a constant part, and another part with an exponential decay and a term that is a polynomial decay, known as Khalfin term. But the Khalfin term is very difficult to be detected, and for this reason it can be neglected [5]. So, we obtain

$$
\left(\rho(t) \mid O_{R}\right) \cong\left(\rho_{R *} \mid O_{R}\right)+\sum_{j} a_{j}(t) e^{-\gamma_{j} t}
$$

where $\rho_{R *}$ is the equilibrium state, $\gamma_{j}$ are the imaginary part of the poles and functions $a_{j}(t)$ depends on the observable $O_{R}$ and the initial condition. 


\section{A dynamical reformulation of quantum logic}

The discussion posed in this paper calls for a reformulation of the quantum logical formalism in order to take into account the dynamical evolution of logics. In order to achieve this aim, we will modify the notion of projective valued measure presented in Section 2 (cf. Eqn. 2). Equation (11) gives us an important clue: in such a decomposition, a one dimensional vector is assigned to a complex number. In the standard formalism of quantum mechanics (for discrete spectra), a vector is assigned to each real number in the spectra. This analogy calls for a generalization of the notion of projection valued measure. We define a complex projection valued measure (CPVM) as a map from the Borel sets of the complex plane $B(\mathbb{C})$ to the set of projection operators satisfying

$$
\begin{array}{r}
P^{\mathbb{C}}: B(\mathbb{C}) \rightarrow \mathscr{P}(\mathscr{H}) \\
\text { such that } \\
P^{\mathbb{C}}(\emptyset)=0 \\
P^{\mathbb{C}}(\mathbb{C})=\mathbf{1} \\
P^{\mathbb{C}}\left(\cup_{j}\left(B_{j}\right)\right)=\sum_{j} P^{\mathbb{C}}\left(B_{j}\right),
\end{array}
$$

for any disjoint denumerable family $B_{j}$. Also,

$$
P^{\mathbb{C}}\left(B^{c}\right)=\mathbf{1}-P^{\mathbb{C}}(B)=\left(P^{\mathbb{C}}(B)\right)^{\perp}
$$

Eqns. (24) constitute a natural generalization of the notion of PVM (Eqns. (2)) to the field of complex numbers. In this way, we postulate that to each non-Hermitian operator $H$ of physical interest we can a assign a CPVM $P_{H}^{\mathbb{C}}$.

In Section 2 we discussed the interpretation of the empirical propositions defined by the spectral theorem in the context of the standard formalism of quantum mechanics. Now we ask for an interpretation of the logical propositions defined by the notion of CPVM associated to a non-Hermitian operator. This can be done in a natural way as follows. Consider a non-Hermitian Hamiltonian $H$ (such as the one in Eqn. (11)) and a region $R_{a, b, c, d}$ of the complex plane defined by

$$
\begin{aligned}
& a \leq \mathfrak{R}(z) \leq b \\
& c \leq \mathfrak{I}(z) \leq d
\end{aligned}
$$

$R_{a, b, c, d}$ is just the cartesian product between the Borel sets $(a, b)$ and $(c, d)$. Now, consider all the complex eigenvalues $Z_{i}\left(R_{a, b, c, d}\right)$ of $H$ which lie inside this region and the associated projection operators $P_{i}^{z_{i}}$. The CPVM $P_{H}^{\mathbb{C}}$ assigns to $R_{a, b, c, d}$ a subspace of $\mathscr{H}_{\mathscr{R}}$ formed by the direct sum of all subspaces associated to each complex eigenvalue: $P_{H}^{\mathbb{C}}\left(R_{a, b, c, d}\right)=\sum_{Z_{i} \in R_{a, b, c, d}} P_{i}^{z_{i}}$.

In order to give a physical interpretation to the above projections as propositions, let us consider the case in which the region involved contains just one eigenvalue 
$z_{j}$ with associated eigenprojetor $P_{j}^{z_{j}}$. If $z_{j}=a_{j}+b_{j} i$, this proposition is naturally interpreted as: "the observed value of $H$ is $a_{j}$ and the decaying rate associated to this mode is $b_{j}$ ". All elementary propositions are of this form. The remainder propositions are naturally formed by direct sums and orthogonal complements of the elementary ones. The notions of conjunction, disjunction and orthogonal complement are just the same as in the standard quantum logical approach. In this way, we reach a (non-Boolean) lattice of projection operators associated to the algebra of non-Hermitian operators. We call this logic $\mathscr{L}^{\mathbb{C}}$. Alike the standard interpretation of the propositions in the von Neumann lattice of projection operators $\mathscr{L}$, which could be termed "static", the propositions of $\mathscr{L}^{\mathbb{C}}$ are interpreted as observed values and decaying rates. In this way, we reach a dynamical version of the of the quantum logical approach.

It is important to remark that our approach allows for a novel quantum logical perspective on the quantum to classical transition. Let us nw turn into the formal aspects of this dynamics. Suppose that the system is initially described by a set observables $\mathbf{V}_{0}$ generating a von Neumann algebra $\mathscr{V}_{0}$ (the minimal von Neumann algebra containing $\mathbf{V}_{0}$ ). To fix ideas, think of $\mathscr{V}_{0}$ as the algebra of bounded observables $\mathscr{B}(\mathscr{H})$ for a Hilbert space $\mathscr{H}$; but notice that in principle, it could be a more general von Neumann algebra, i.e., a Type II or Type III factor (depending on the model that we are studying). Under the action of the non-Hermitian evolution $U(t)=\exp ^{-i H t}, \mathbf{V}_{0}$ is mapped to the set

$$
\mathbf{V}_{t}=U(t) \mathbf{V}_{0} U^{\dagger}(t)
$$

As before, $\mathbf{V}_{t}$ generates an algebra $\mathscr{V}_{t}$. In this way, the classical limit will be reached if the limiting algebra $\mathscr{V}_{\infty}$ is a Boolean one. Notice that the formulation of this problem is plagued of subtleties: the question about under which conditions the classical limit is reached can be a hard mathematical problem, which goes far from the scope of this article. Notwithstanding, the examples presented in previous Sections show us that this picture works for cases of interest.

If the process is suitably defined, Eqn. (26) generates a family of von Neumann algebras

$$
\mathscr{F}_{\mathscr{V}_{0}}=\left\{\mathscr{V}_{t}\right\}_{t \in \mathbb{R}}
$$

Notice also that each von Neumann algebra $\mathscr{V}_{t}$ has associated an orthomodular lattice [17] of projection operators $\mathscr{L}_{y_{t}}$. In this way we also have the family of quantum logics

$$
\mathscr{F}_{\mathscr{L}_{0}}=\left\{\mathscr{L}_{\mathscr{V}_{t}}\right\}_{t \in \mathbb{R}}
$$

which allow for a quantum logical description of the classical limit process. Notice that the family $\mathscr{F}_{\mathscr{L}_{0}}$ constitutes a strain of orthomodular lattices, by appealing to the non-Hermitian operator responsible of the non-unitary evolution. If the classical limiting process is successful, the orthomodular lattice $\mathscr{L}_{\mathscr{V}_{\infty}}$ will be a Boolean 
one. If we now consider the category of von Neumann algebras and the category of orthomodular lattices, we find a map $\Phi$ relating them as follows

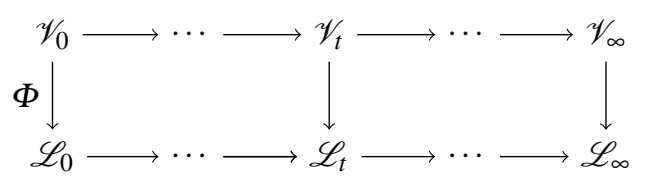

It is worth mention again that a structure such as the one described in the above diagram cannot be included in the standard unitary formulation of quantum mechanics.

\section{Conclusions}

In this paper we addressed the question of how to study the dynamical evolution of quantum logics associated to quantum systems reaching the classical limit. With this aim we have presented a quantum logical approach to the formulation of quantum mechanics based on non-Hermitian operators. This allows us to describe the time evolution of algebras which, being initially non-commutative, become Boolean ones because of the action of a non-unitary time evolution.

This novel perspective allows to describe a family of algebras evolving in time. The starting algebra can be non-Boolean, while the final one (for infinite time) will be Boolean. In this way we presented a quantum logical approach for the reaching of the classical limit, something which was not present in the previous literature. We have shown in Section 3 that this was difficult to describe using the standard formalism of quantum mechanics, and thus, a shift to the non-Hermitian Hamiltonians approach was in order. We have described concrete examples of this transition between logics in Sections 4, 5 and 6.

In Section 7 we have introduced the novel notion of complex projective valued measure (Eqn. (24)), a generalization of the standard notion (Eqn. (2)) to the field of complex numbers. This allows us to reinterpret the results of previous Sections with a new quantum logical formalism prepared to deal with decaying times associated to physical processes. In this way, we built a family of orthomodular lattices continuously parameterized by time, for which the initial lattice is fully quantum mechanical, but the limiting one is Boolean.

We hope that the ideas presented in this paper can be further developed in order to study the formal aspects of this algebraic approach to decoherence and the classical limit. This could find applications in theories more general than standard quantum theory, such as algebraic relativistic quantum field theory and algebraic quantum statistical mechanics [49]. 


\section{References}

1. F. G. Scholtz, H.B. Geyer and F.J.W. Hahne, Annals Of Physics, 213, 74-101, (1992).

2. A. Guo, G.J. Salamo, D. Duchesne, R. Morandotti, M. Volatier-Ravat, V. Aimez, G. A. Siviloglou and D. N. Christodoulides, Physic. Rev. Lett., 103, 093902, (2009).

3. S. Klaiman, U. Günther and N. Moiseyev, Physic. Rev. Lett., 101, 080402, (2008).

4. O. Bendix, R. Fleischmann, T. Kottos and B. Shapiro, Jour. Phys. A: Math. Theo., 43, (2010).

5. M. Castagnino and S. Fortin, Jour. of Phys. A 45, 444009 (2012).

6. M. Castagnino, R. Id Betan, R. Laura and R. J. Liotta, J. Phys. A: Math. Gen., 35, 6055-6074, 2002.

7. A. Bohm, Quantum mechanics, foundations and applications, Springer Verlag, Berlin, 1986.

8. A. Bohm and N. L. Harshman, "Quantum theory in the rigged hilbert space - Irreversibility from causality", in A. Bohm, H. Doebner and P. Kielanowski (Eds.), Irreversibility and Causality Semigroups and Rigged Hilbert Spaces, Volume 504 of the series Lecture Notes in Physics, Springer Verlag, Berlin, pp 179-237, 2007.

9. R. Weder, Jour. Math. Phys., 15, 20, 1974.

10. E. Sudarsham, C. Chiu and V. Gorini, Phys. Rev. D 18, 2914-2929 (1978).

11. M. Castagnino and R. Laura, Phys. Rev. A 56, 108-119 (1997).

12. R. Laura and M. Castagnino, Phys. Rev. A 57, 4140-4152 (1998).

13. R. Laura and M. Castagnino, Phys. Rev. E 57, 3948-3961 (1998).

14. M. Castagnino and S. Fortin, Mod. Phys. Lett. A 26, 2365-2373 (2011).

15. M. Castagnino and S. Fortin, Jour. Phys. A 45, 444009 (2012).

16. J. Lacki (2000). "The Early Axiomatizations of Quantum Mechanics: Jordan, von Neumann and the Continuation of Hilbert's Program." Archive for History of Exact Sciences, 54: 279318.

17. M. Rèdei (1998), Quantum Logic in Algebraic Approach, Dordrecht: Kluwer Academic Publishers.

18. M. Reed and B. Simon (1972). Methods of modern mathematical physics I: Functional analysis, New York: Academic Press.

19. J. von Neumann (1996). Mathematical Foundations of Quantum Mechanics, Princeton: Princeton University Press.

20. G. Birkhoff and J. von Neumann, Annals of Math. 37, 823-843 (1936).

21. Handbook Of Quantum Logic And Quantum Structures (Quantum Logic), Edited by K. Engesser, D. M. Gabbay and D. Lehmann, North-Holland (2009).

22. Kalmbach (1983). Orthomodular Lattices, San Diego: Academic Press.

23. M. L. Dalla Chiara, R. Giuntini and R. Greechie (2004), Reasoning in Quantum Theory, Dordrecht: Kluwer Academic Publishers.

24. C. Piron (1976). Foundations of Quantum Physics, Cambridge: Addison-Wesley.

25. V. Varadarajan (1968). Geometry of Quantum Theory I, Princeton: van Nostrand.

26. V. Varadarajan (1970). Geometry of Quantum Theory II, Princeton: van Nostrand.

27. I. Stubbe and B.V. Steirteghem (2007). "Propositional systems, Hilbert lattices and generalized Hilbert spaces.” In Handbook Of Quantum Logic Quantum Structures: Quantum Structures, K. Engesser, D.M. Gabbay and D. Lehmann (eds.), 477-523. Amsterdam: Elsevier.

28. M. Solèr, Communications in Algebra 23, 219-243.

29. M. Schlosshauer (2007). Decoherence and the Quantum-to-Classical Transition, Berlin: Springer.

30. C. Gardiner and P. Zoller (2004). Quantum noise, Springer Verlag.

31. S. Fortin and L. Vanni, Found. of Phys. 44, 1258-1268 (2014).

32. W. Zurek, Phys. Rev. D 24, 1516-1525 (1981).

33. W. Zurek, Rev. of Mod. Phys. 75, 715-776 (2003).

34. M. Castagnino, S. Fortin, R. Laura and O. Lombardi, Class. and Quant. Grav. 25, 154002 (2008).

35. M. Castagnino, S. Fortin and O. Lombardi, Mod. Phys. Lett. A 25, 1431-1439 (2010).

36. M. Castagnino, S. Fortin and O. Lombardi, Jour. of Phys. A 43, 065304 (2010). 
37. M. Castagnino, S. Fortin and O. Lombardi, Mod. Phys. Lett. A 25, 611-617 (2010).

38. S. Ardenghi, S. Fortin, M. Narvaja and O. Lombardi, Int. Jour. of Mod. Phys. D 20, 861-875 (2011).

39. M. Castagnino and S. Fortin, Int. Jour. of Theor. Phys. 50, 2259-2267 (2011).

40. M. Castagnino and S. Fortin, Int. Jour. of Theor. Phys. 52, 1379-1398 (2013).

41. S. Fortin, O. Lombardi and M. Castagnino, Braz. Jour. of Phys. 44, 138-153 (2014).

42. M. Castagnino and S. Fortin, Mod. Phys. Lett. A 26, 2365-2373 (2011).

43. J. Bub (1997). Interpreting the Quantum World, Cambridge: Cambridge University Press.

44. D. W. Cohen (1989). An introduction to Hilbert space and quantum logic, New York: Springer.

45. C. Kiefer and D. Polarski, Adv. Science Lett. 2, 164-173 (2009).

46. C. Kiefer and D. Polarski, Annalen Phys. 7, 137-158 (1998).

47. M. Castagnino and R. Laura, Phys. Rev. A 62, 022107 (2000).

48. M. Castagnino and O. Lombardi, Phys. Rev. A 72, 012102 (2005).

49. M. Rédei and S. Summers, Studies in History and Philosophy of Science Part B: Studies in History and Philosophy of Modern Physics Volume 38, Issue 2, (2007) 390-417.

50. M. Castagnino, M. Gadella, F. Gaioli and R. Laura, Int. Jour. of Theor. Phys. 38, 2823-2865 (1999).

51. M. Castagnino, R. Id Betan, R. Laura and R. J. Liotta, Jour. of Phys. A 35, 6055 (2002).

52. M. Castagnino and R. Laura, Phys. Rev. A 56, 108 (1997).

53. A. Bohm, I. Antoniou and P. Kielanowski, J. Math. Phys. 36, 2593 (1995).

54. A. Bohm, M. Gadella and G. Bruce Mainland, Am. J. Phys. 57, 1103 (1989).

55. I.E. Antoniou, M. Gadella, E. Karpov, I. Prigogine, G. Pronko, Chaos, Solitons \& Fractals 12, 2757-2775 (2001).

56. Baumgärtel, Rev. Math. Phys. 18, 61 (2006).

57. T. Petrosky, I. Prigogine and S. Tasaki, Physica A 173, 175-242 (1991).

58. T. Petrosky and I. Prigogine, Physica A 175, 146-209 (1991).

59. I. Antoniou and I. Prigogine, Physica A 192, 443-464 (1993).

60. I.E. Antoniou, Z. Suchenecki, R. Laura and S. Tasaki, Physica A 241, 737-772 (1997).

61. J. M. Jauch (1968). Foundations of Quantum Mechanics, Cambridge: Addison-Wesley. 